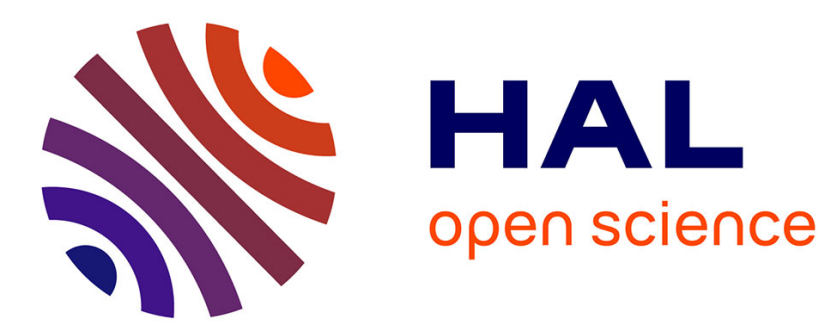

\title{
La science juridique entre politique et sciences humaines (XIXème-XXème siècles)
}

\author{
Frédéric Audren, Jean-Louis Halpérin
}

\section{To cite this version:}

Frédéric Audren, Jean-Louis Halpérin. La science juridique entre politique et sciences humaines (XIXème-XXème siècles). Revue d'histoire des sciences humaines, 2001, 4, pp.3 - 7. hal-01462082

\section{HAL Id: hal-01462082 \\ https://hal-sciencespo.archives-ouvertes.fr/hal-01462082}

Submitted on 8 Feb 2017

HAL is a multi-disciplinary open access archive for the deposit and dissemination of scientific research documents, whether they are published or not. The documents may come from teaching and research institutions in France or abroad, or from public or private research centers.
L'archive ouverte pluridisciplinaire HAL, est destinée au dépôt et à la diffusion de documents scientifiques de niveau recherche, publiés ou non, émanant des établissements d'enseignement et de recherche français ou étrangers, des laboratoires publics ou privés. 


\section{La science juridique entre politique et sciences humaines (XIX ${ }^{\text {ème }}-X^{\text {ème }}$ siècles)}

Science historique, science morale et politique, science sociale... Ces expressions ont été utilisées pour l'étude du droit depuis plusieurs siècles. L'idée de joindre le droit à l'histoire a fleuri au XVI ${ }^{\text {ème }}$ siècle, dans le milieu des humanistes français de l'École de Bourges étudié par Donald R. Kelley. Avec les Lumières le droit trouve sa place dans les sciences morales et politiques, toujours en étroit contact avec l'histoire, dans le sillage de Montesquieu. Sous la Révolution, les Idéologues, comme Daunou ou Destutt de Tracy, envisagent de créer des écoles supérieures de sciences morales et politiques où serait enseignée la législation. Le schéma d'organisation de l'Institut répond à cette classification. Et l'expression «science sociale » est déjà appliquée au droit par des juristes de la première moitié du XIX ${ }^{\text {ème }}$ siècle, à l'instar de Cabantous en 1839, à une époque où Jean-Baptiste Duvergier écrit que « l'influence de la société sur la législation et l'action de la législation sur la société sont également incontestables ». Pourtant, en dépit de ces rapprochements précoces, la science juridique a pendant des siècles cultivé l'isolement: dans toute le continent européen, sur la base de la compilation de Justinien redécouverte à la fin du $\mathrm{XI}^{\text {ème }}$ siècle - avec l'élaboration d'une «Écriture du droit en une pratique nécessairement tautologique et sui-référentielle » (Yan Thomas) - puis particulièrement en France avec l'avènement de la codification napoléonienne qui fait table rase des sources de l'ancien droit et s'accompagne de la mise en place d'écoles de droit fermées sur elles-mêmes, destinées à former des praticiens par la seule connaissance du Code, sans recours aux réflexions historiques ou philosophiques. Ce sentiment de singularité du Droit par rapport aux autres disciplines s'est prolongé jusqu'à nos jours: dans les réticences des juristes à faire entrer la sociologie ou la linguistique dans leurs facultés, dans les relations toujours difficiles entre historiens-juristes et historiens littéraires depuis les rendez-vous manqués (ou limités à quelques exceptions comme Gabriel Le Bras) avec les Annales, dans un discours formaliste, se proclamant positiviste, voire normativiste, qui affirme « l'autonomie absolue de la forme juridique par rapport au monde social » (Pierre Bourdieu) et la séparation entre la science du droit et la connaissance des réalités sociales. Un tel isolationnisme explique la pauvreté de l'historiographie des disciplines juridiques en France jusqu'à ces dernières décennies, y compris de la part des historiens du droit, comme s'en plaignait Jacques Poumarède en 1980.

Dans le cadre d'un regain d'intérêt pour l'histoire de l'enseignement du droit - dont témoigne la Revue d'histoire des facultés de droit et de la science juridique depuis 1984 -, pour l'histoire de la science juridique française et surtout allemande (notamment Olivier Beaud, Carlos Miguel Herrera ou encore Olivier Motte), d'une curiosité avivée des chercheurs en sciences humaines pour un champ juridique réputé en expansion dans les sociétés post-modernes, ce numéro propose une série de réflexions sur les rapports entre droit et sciences humaines sous le titre La science juridique entre politique et sciences humaines. L'histoire sert de lien à ces analyses, dans la mesure où elle a joué un rôle de " pont» ou de «fenêtre » entre le droit et les sciences 
humaines, en tant que discours hétérogène, comme discipline spécifique ou au titre de la défense de la tradition face aux « dangereuses innovations ». L'ouverture du droit à l'histoire, avec tout ce qu'elle suppose en termes de signification politique, est ici abordée à travers une série d'assauts ou de chocs venus du dehors ou ressentis comme tels par les juristes confrontés aux démarches scientifiques des non-juristes.

Dans l'ordre chronologique, Georges Navet, qui a exploré «l'usage de Vico en France » dans sa thèse de 1987 (thèse de science politique, ce qui est révélateur), s'intéresse à l'œuvre d'Eugène Lerminier (1803-1857). Un des introducteurs des thèses de Vico et de Savigny en France, Lerminier a occupé une position singulière dans l'Université française (comme professeur de législations comparées au Collège de France) et joué un rôle déterminant par ses travaux dans le développement des études historiques du droit et dans la réflexion sur l'articulation entre droit et philosophie. N'écrit-il pas, en 1829, que « le droit est une science morale qui vient entre la philosophie et l'histoire »? En contact un moment avec les saint-simoniens, partie prenante dans la discussion autour de Vico menée par Michelet et Victor Cousin, Lerminier révèle les orientations nouvelles de la petite troupe de juristes français (dont le premier noyau s'est formé autour de la revue Thémis d'Athanase Jourdan) qui ont voulu s'affranchir, dans la première moitié du XIX ${ }^{\text {ème }}$ siècle, de la méthode exégétique régnant en maître sur les facultés de droit. Il témoigne en même temps des faiblesses d'une démarche qui privilégie la perspective philosophique et l'histoire de l'idée de droit sur l'étude des institutions, telle que commencent à la pratiquer ses contemporains Henri Klimrath (1807-1837), Firmin Laferrière (1798-1861) et Edouard-René Laboulaye (1811-1883).

Jean-Louis Halpérin, qui s'était déjà penché sur l'œuvre de l'historien du droit Adhémar Esmein (1848-1913), situe la reconnaissance de l'histoire du droit comme discipline dans son contexte européen: une trajectoire relativement complexe, qui à la fois réalise et trahit les ambitions de l'École historique du droit de Savigny pour aboutir à une consécration universitaire rapidement entamée par un repli et une crise d'identité. Du discours historique à l'histoire du droit érudite, la question de la « méthode historique » traverse tout le XIX ${ }^{\text {ème }}$ siècle.

Elle est encore présente chez les juristes français dans les années 1900. Guillaume Sacriste a pris l'exemple de Maurice Deslandres (1862-1932), professeur à la Faculté de droit de Dijon, auteur d'un article programmatique sur la crise de la science politique en France. La méthode historique a souvent été invoquée par les « rénovateurs » de la science juridique française, Saleilles et Gény, que Deslandres a bien connus à Dijon. L'histoire est alors sollicitée, face au défi posé au droit par la sociologie naissante. La concurrence entre juristes et sociologues, objet de travaux récents comme ceux de Francine Soubiran-Paillet, s'exerce particulièrement sur le terrain de l'histoire du droit.

Un dernier jalon est envisagé autour de la création de l'agrégation de science politique en 1971, sujet de la contribution de Marc Milet, auteur d'une thèse récente sur les engagements politiques des juristes français de 1914 à 1995 . La science politique a en commun avec l'histoire du droit le statut d'une « petite » discipline avec son corps spécifique d'enseignants. L'établissement d'une agrégation de science politique, 75 ans après la création de celle d'histoire du droit, montre à nouveau les enjeux scientifiques de l'ouverture du droit à d'autres savoirs dans le contexte de l'après-mai 1968 (avec un ministre, Edgar Faure, qui était aussi professeur d'histoire du droit). 
Ces quatre articles sont complétés par l'évocation - à partir de documents inédits réunis par Frédéric Audren et Georges Navet - de la carrière de Lerminier au Collège de France (1831-1849), par la publication de quelques lettres, conservées au Collège de France, adressées à Marcel Mauss par l'historien du droit Paul Huvelin (18731924) ainsi que la réédition d'un article peu connu de ce dernier, «Les cohésions humaines. La place qu'y tiennent la contrainte juridique et l'attraction morale » (1923). Très influencé par Durkheim, Huvelin y présente sa conception de la sociologie historique du droit. Ils sont, avec son ami Emmanuel Lévy (1871-1943), professeur de droit civil, les seuls représentants de la faculté de droit au sein de L'Année sociologique.

Politiques de l'histoire, aurions-nous pu intituler ce numéro, car la dimension politique de cette histoire s'impose à tout chercheur. Elle est omniprésente dans les contributions présentées, qu'il s'agisse du positionnement politique des juristes étudiés ou des enjeux de pouvoir des batailles universitaires suscitées par l'histoire du droit ou la science politique. Selon la terminologie de Pierre Bourdieu, le droit est ici envisagé comme un " univers social spécifique », un champ dans lequel se déploient des luttes d'influence. Dans un groupe social réputé conservateur - celui des juristes et plus étroitement des professeurs de droit - les partisans d'une ouverture à l'histoire et aux sciences humaines ont pu apparaître comme des « trublions », contestataires de l'ordre juridique établi. Il a existé une veine libérale de l'histoire du droit - avec Lerminier et Laboulaye - une veine républicaine avec Esmein. Pour autant, il ne faut pas négliger la fonction légitimante du discours historique et son emploi massif par des théoriciens conservateurs depuis Savigny jusqu'aux partisans des dictatures du XX ${ }^{\text {ème }}$ siècle, Leicht en Italie, Eckhardt en Allemagne et Français Olivier-Martin (1879-1952), chantre du corporatisme et ferme soutien du régime de Vichy. Guillaume Sacriste soupçonne l'usage de l'histoire par Deslandres ou Gény d'être inséparable de présupposés réactionnaires. Le débat reste ouvert en présence de juristes-historiens ou sociologues plus portés à favoriser le changement social. Comme le montre la complexité d'un itinéraire intellectuel comme celui de Saleilles, récemment restitué par un article majeur de Paolo Grossi, la grille de lecture politique ne suffit pas, selon nous, à rendre compte de tous les aspects de ces rapports tumultueux entre droit et sciences humaines. Encore aujourd'hui les théoriciens du droit s'interrogent, au-delà des clivages politiques, sur la portée de l'historicité des phénomènes juridiques. Si l'accord règne sur l'inscription du droit dans le temps et même dans la longue durée - « par nature, le droit dépend de son application répétée » écrit Reinhart Koselleck - la controverse n'a pas cessé depuis que Kelsen a entendu, dès son livre de 1911 Hauptprobleme der Staatsrechtslehre, séparé radicalement la théorie pure du droit, positiviste, descriptive et normativiste des approches historiques ou sociologiques, dont il ne contestait pas l'intérêt, mais qu'il rejetait hors du droit et des sciences normatives du Sollen (devoir-être), dans le domaine des sciences sociales et causales du Sein (être). L'histoire du droit, conçue à la fois comme une sociologie historique et une histoire culturelle de la pensée juridique, peut-elle prétendre expliquer le droit, au delà de la compréhension du processus de production des règles juridiques ou se heurte-t-elle à la dimension fondamentalement logique et discursive des normes juridiques ? Dans l'enseignement des facultés de droit, comme dans l'appréhension de l'essence de l'interprétation du droit (Gadamer), les juristes restent irrémédiablement tiraillés entre Dogmatique et Histoire, entre formalisme et socio- 
logisme, entre le ralliement aux sciences humaines au nom d'une conception du droit comme « reflet du social » et défense de l'autonomie de leur discipline en raison de sa spécificité et de sa complexité.

\author{
Frédéric AUDREN \\ Université Paris II-Panthéon-Assas \\ audren@ccr.jussieu.fr \\ et \\ Jean-Louis HALPÉRIN \\ Université de Bourgogne \\ Centre Georges Chevrier (CNRS/UMR 5605) \\ jean-louis.halperin@u-bourgogne.fr
}

\title{
Bibliographie
}

AudRen F., (ed.), 2000, Socialismes des juristes, Cahiers Trimestriels Jean Jaurès, avril-juin, 156 (numéro spécial).

ARNAUD A.J., 1975, Les juristes face à la société du XIX'me siècle à nos jours, Paris, PUF.

BEAUD O., WACHSMANN P., (ed.), 1997, La science juridique française et science juridique allemande de 1870 à 1918, Strasbourg, Presses Universitaires de Strasbourg.

BouRdieu P., 1986, La force du droit. Éléments pour une sociologie du champ juridique, Actes de la Recherche en Sciences Sociales, 64, 3-19.

BÖCKENFÖRDE E.W., 2000 (1965), L'École historique du droit et le problème de l'historicité du droit, Le Droit, l'État et la Constitution Démocratique, Paris, LGDJ-Bruylant, 53-76.

BÜRGE A., 1991, Das französische Privatrecht im 19. Jahrundert. Zwischen Tradition und Pandektenwissenschaft, Liberalismus und Etatismus, V. Klostermann, Frankfurt-am-Main.

CHAZEL F., 1991, Émile Durkheim et l'élaboration d'un programme de recherche en sociologie du droit, in Chazel F., Commaille J., (ed.), Normes juridiques et régulation sociale, Paris, LGDJ, 27-38.

DIDRY C., 1990, De l'État aux groupes professionnels. Les itinéraires croisés de L. Duguit et É. Durkheim au tournant du siècle (1880-1900), Genèses. Sciences Sociales et Histoire, décembre, 2, 5-27.

DIDRY C., 2000, La réforme des groupements professionnels comme expression de la conception durkheimienne de l'État, Revue Française de Sociologie, 41-3, 513-538.

Gadamer H.G., 1976, Vérité et méthode, Paris, Éditions du Seuil.

Grossi P., 1993, Assolutismo giuridico e diritto privato. Lungo l'itinarario scientifico di Raymond Saleilles, Rivista di Diritto Civile, XXXIX-3, 345-398.

HALPÉRIN J.L., 1997, Adhémar Esmein et les ambitions de l'histoire du droit, Revue Historique de Droit Français et Étranger, 75-3, 415-433.

Herrera C.M., 1997, Théorie juridique et politique chez Hans Kelsen, Paris, Kimé.

Kelley D.R., 1970, Foundations of Modern Historical Scholarship, New York-London, Columbia University Press.

Kelley D.R., 1984, Historians and the Law in Postrevolutionary France, Princeton, Princeton University Press.

KelSEN H., 1923, Hauptprobleme der Staatsrechtslehre, Tübingen, J.C.B. Mohr. 
Koselleck R., 1997, L'expérience de l'histoire, Paris, Seuil-Gallimard.

Lascoumes P., 1991, Le droit comme science sociale, in Chazel F., Commaille J., (ed.), Normes juridiques et régulation sociale, Paris, LGDJ, 39-49.

Lenoir R., 1993, Le droit et ses usages, in Besnard Ph., Borlandi M., Vogt P., (ed.), Division du travail et lien social. Durkheim un siècle après, Paris, PUF, 165-183.

LETERRIER S.A., 1995, L'Institution des sciences morales. L'Académie des sciences morales et politiques (1795-1850), Paris, l'Harmattan.

Milet M., 2000, Les professeurs de droit citoyens. Entre ordre juridique et espace public. Contribution à l'étude des interactions entre les débats et les engagements des juristes français (1914-1995), thèse pour le doctorat de science politique, Université Paris IIPanthéon-Assas (3 volumes).

MоттE O., 1989-1990, Lettres inédites de juristes français du XIX'me siècle conservées dans les archives et bibliothèques allemandes, Bonn, Bouvier (2 volumes).

Navet G., 1987, De l'usage de Vico en France : le problème de la légitimité du droit civil, thèse de l'Université de Reims.

Poumarede J., 1980, Pavane pour une histoire du droit défunte. Sur un centenaire oublié, Procès. Cahiers d'Analyse Politique et Juridique, 6, 91-102.

RÉMY Ph., 1987, La Thémis et le droit naturel, Revue d'Histoire des Facultés de Droit et de la Science Juridique, 4, 145-160.

Soubiran-PAillet F., 1997, Histoire du droit et sociologie: interrogations sur un vide disciplinaire, Genèses, Histoire et Sciences Sociales, 29, 141-163.

SOUBIRAN-PAILlET F., 2000, Juristes et sociologues français d'après-guerre : une rencontre sans lendemain, Genèses. Histoire et sciences sociales, 41, 125-142.

Thomas Y., 1986, Droit, in Burguiere A., (ed. ), Dictionnaire des sciences historiques, Paris, PUF, 205-212.

Thommasset C., Vanderlinden J., Jestaz Ph., (ed.), 2000, François Gény, Mythes et réalités, Québec, Yvon Blais-Dalloz-Bruylant.

WeISZ G., 1979, L'idéologie républicaine et les sciences sociales. Les durkheimiens et la chaire d'histoire d'économie sociale à la Sorbonne, Revue Française de Sociologie, 20, 83-112. 